\title{
Hamid Haddadou(i) \\ H-convergence of a class of quasilinear equations in perforated domains beyond periodic setting
}

Received: 9 September 2020 / Accepted: 18 January 2021 / Published online: 6 February 2021

(C) The Author(s) 2021

\begin{abstract}
In this paper, we aim to study the asymptotic behavior (when $\varepsilon \rightarrow 0$ ) of the solution of a quasilinear problem of the form $-\operatorname{div}\left(A^{\varepsilon}\left(\cdot, u^{\varepsilon}\right) \nabla u^{\varepsilon}\right)=f$ given in a perforated domain $\Omega \backslash T_{\varepsilon}$ with a Neumann boundary condition on the holes $T_{\varepsilon}$ and a Dirichlet boundary condition on $\partial \Omega$. We show that, if the holes are admissible in certain sense (without any periodicity condition) and if the family of matrices $(x, d) \mapsto A^{\varepsilon}(x, d)$ is uniformly coercive, uniformly bounded and uniformly equicontinuous in the real variable $d$, the homogenization of the problem considered can be done in two steps. First, we fix the variable $d$ and we homogenize the linear problem associated to $A^{\varepsilon}(\cdot, d)$ in the perforated domain. Once the $H^{0}$-limit $A^{0}(\cdot, d)$ of the pair $\left(A^{\varepsilon}, T^{\varepsilon}\right)$ is determined, in the second step, we deduce that the solution $u^{\varepsilon}$ converges in some sense to the unique solution $u^{0}$ in $H_{0}^{1}(\Omega)$ of the quasilinear equation - $\operatorname{div}\left(A^{0}\left(\cdot, u^{0}\right) \nabla u\right)=\chi^{0} f$ (where $\chi^{0}$ is $L^{\infty}$ weak ${ }^{\star}$ limit of the characteristic function of the perforated domain). We complete our study by giving two applications, one to the classical periodic case and the second one to a non-periodic one.
\end{abstract}

Mathematics Subject Classification $35 \mathrm{~B} 40 \cdot 35 \mathrm{~B} 27 \cdot 35 \mathrm{~J} 62$

\section{Introduction}

The main goal of this work is to give, in the framework of the $H^{0}$-convergence notion (the generalization of the $H$-convergence to perforated domains), a general homogenization result of a type of quasilinear equations with a mixed Neumann-Dirichlet boundary conditions, beyond the periodic setting. More precisely, we study the asymptotic behaviour of the solution of the following problem:

$$
\left\{\begin{array}{l}
-\operatorname{div}\left(A^{\varepsilon}\left(\cdot, u^{\varepsilon}\right) \nabla u^{\varepsilon}\right)=f \quad \text { in } \Omega \backslash T_{\varepsilon}, \\
A^{\varepsilon}\left(\cdot, u^{\varepsilon}\right) \nabla u^{\varepsilon} \cdot v=0 \text { on } \partial T_{\varepsilon}, \\
u^{\varepsilon}=0 \text { on } \partial \Omega
\end{array}\right.
$$

where $\Omega$ is a bounded open subset of $\mathbb{R}^{n},\left\{T_{\varepsilon}\right\}$ is sequence of compact subsets of $\Omega$, not necessarily periodically distributed, and where $f \in L^{2}(\Omega), A^{\varepsilon}:(x, d) \in(\Omega, \mathbb{R}) \longmapsto A^{\varepsilon}(x, t) \in \mathbb{R}^{n \times n}$ is a sequence of Caratheodory functions uniformly coercive, uniformly bounded and uniformly equicontinuous matrix fields in the variable $d$. We show that, under a suitable conditions on the equicontinuity modulus and $L^{p}$-estimate assumption, there exists a subsequence of $\varepsilon$ (still denoted by $\varepsilon$ ), a positive function $\chi^{0} \in L^{\infty}(\Omega)$ and a matrix field $A^{0}(\cdot, \cdot)$ which satisfies the same properties as $A^{\epsilon}(\cdot, \cdot)$ such that $\chi^{\varepsilon} \rightarrow \chi^{0}$ weakly ${ }^{\star}$ in $L^{\infty}(\Omega)$,

$$
\left(A^{\varepsilon}(\cdot, d), T_{\varepsilon}\right) \stackrel{H^{0}}{\rightarrow} A^{0}(\cdot, d) \quad \text { in } \Omega, \forall d \in \mathbb{R}^{n},
$$

H. Haddadou $(\varangle)$

LCSI laboratory, Ecole netionale Suprieure d'Informatique (ESI ex INI), Algiers, Algeria

E-mail: h_haddadou@esi.dz 
and, if we denote by $\sim$ the extension by 0 from $\Omega_{\varepsilon}$ to $\Omega$, we have

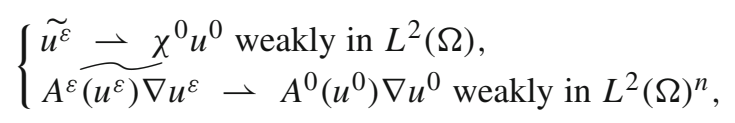

where $u^{0}$ is the solution of

$$
\left\{\begin{array}{l}
-\operatorname{div}\left(A^{0}\left(\cdot, u^{0}\right) \nabla u^{0}\right)=\chi^{0} f \text { in } \Omega \\
u^{0}=0 \text { on } \partial \Omega
\end{array}\right.
$$

We complete our study by giving two applications of the established compactness results. The first application is for the classical periodic case, where the obtained result coincides (in our framework) with a result given in [7]. While, the second one which concerns a non-periodic case introduced in [5] is an original result.

Our work generalizes that of Murat-Bocardo given in [4] which treated in the general framework of $\mathrm{H}$ convergence the same type of quasilinear equations in fixed domains without holes. The periodic case with Lipschitz continuous coefficients was subsequently processed by Artola-Duvaut in [1]. On the other hand, for periodically perforated domains, the same type of quasilinear equations was firstly studied in Bendib [2] and Bendib-Tcheugoué Teboué [3], with Lipschitz continuous coefficients and linear Robin conditions. After this Cabarrubias-Donato have studied in [7] this equation with a nonlinear Robin condition boundary of the holes and the module of equicontinuity satisfies a suitable assumption introduced by Chipot in [9], but not assumed to be Lipschitz continuous. For the homogenization of other type of Neumann quasilinear equations in perforated domains with data satisfying a general assumptions of abstract homogenization, see for example $[8,13]$ among others.

This article is organized as follows: Sect. 2 is devoted to some preliminary results on the $H^{0}$-convergence as introduced by [5]. This notion generalizes that of $H$-convergence in fixed domains due to Murat-Tartar (see $[12,14])$. We give at the end of this section, a new result about a pointwise estimate of the dierence of two $H^{0}$-limits. In Sect. 3, we present our main compactness results for a class of quasilinear equations in perforated domains in the general framework of $H^{0}$-convergence. Section 4 is devoted to the proofs of our results. Finally, in Sect. 5, we give two applications of the obtained compactness results, namely the classical periodic case and a certain non-periodic case.

\section{Notations and preliminary results}

\subsection{Notations}

- $\{\varepsilon\}$ denotes a strictly decreasing sequence converging to zero,

- if $\zeta=\left(\zeta_{i}\right)_{1 \leq i \leq n}$ and $\xi=\left(\xi_{i}\right)_{1 \leq i \leq n}$ are two vectors, we set

$$
\zeta \cdot \xi=\sum_{i=1}^{n} \zeta_{i} \xi_{i} \quad \text { and }|\xi|=\left(\sum_{i=1}^{n} \xi_{i}^{2}\right)^{\frac{1}{2}}
$$

- for matrix $A$ in $R^{n \times n}$, we set

$$
|A|=\sup \left\{|A \xi| \text { s.t. }|\xi|=1 \text { and } \xi \in \mathbb{R}^{n}\right\},
$$

- $\chi_{\mathcal{O}}$ denotes the characteristic function of a subset $\mathcal{O}$ of $\mathbb{R}^{n}$,

- for two real numbers $\alpha$ and $\beta$ such that $0<\alpha<\beta, M(\alpha, \beta ; \Omega)$ is the set of the matrix fields $A=$ $\left(A_{i j}\right)_{1 \leq i, j \leq n}$ defined on $\Omega$ such that almost everywhere in $\Omega$, we have

$$
\left\{\begin{array}{l}
\text { (i) } A_{i j} \in L^{\infty}(\Omega), \text { for } i, j=1, \ldots, n \\
\text { (ii) } \alpha|\xi|^{2} \leq A \xi \cdot \xi, \text { for } \xi \in \mathbb{R}^{n} \\
\text { (iii) } A^{-1} \xi \cdot \xi \geq \beta^{-1}|\xi|^{2}, \text { for } \xi \in \mathbb{R}^{n} .
\end{array}\right.
$$




\subsection{Preliminary results on the H-convergence for perforated domains}

Since we work in the framework of the $H^{0}$-convergence, we recall in this subsection some preliminary results about this notion and we give at the end a useful new result on the pointwise estimate of the dierence of two $H^{0}$-limits.

We introduce the perforated domain by

$$
\Omega_{\varepsilon}=\Omega \backslash T_{\varepsilon},
$$

where $\left\{T_{\varepsilon}\right\}$ is a sequence of compact subsets of $\Omega$ and set

$$
V_{\varepsilon}=\left\{v \in H^{1}\left(\Omega_{\varepsilon}\right) \text { s.t. } v=0 \text { on } \partial \Omega\right\} .
$$

We denote by $\sim$ the extension by 0 from $\Omega_{\varepsilon}$ to $\Omega$ and set $\chi^{\varepsilon}=\chi_{\Omega_{\varepsilon}}$. In the following $v$ denotes the outward normal unit vector to the boundary of $\Omega_{\varepsilon}$.

Definition 2.1 ([5]) The sequence $\left\{T_{\varepsilon}\right\}$ is said to be admissible (in $\Omega$ ) if i) every $L^{\infty}$ weak ${ }^{\star}$ limit point of $\left\{\chi^{\varepsilon}\right\}$ is positive almost everywhere in $\Omega$,

ii) there exists a positive real $C$, independent of $\varepsilon$, and a sequence $\left\{P_{\varepsilon}\right\}$ of linear extension operators such that for each $\varepsilon$

$$
\left\{\begin{array}{l}
P_{\varepsilon} \in \mathcal{L}\left(V_{\varepsilon}, H_{0}^{1}(\Omega)\right), \\
\left(P_{\varepsilon} v\right)_{\Omega_{\varepsilon}}=v, \quad \forall v \in V_{\varepsilon}, \\
\left\|\nabla P_{\varepsilon} v\right\|_{L^{2}(\Omega)^{n}} \leq C\|\nabla v\|_{L^{2}\left(\Omega_{\varepsilon}\right)^{n}}, \quad \forall v \in V_{\varepsilon} .
\end{array}\right.
$$

We denote by $P_{\varepsilon}^{\star}$ the adjoint operator of $P_{\varepsilon}$, which is defined from $H^{-1}(\Omega)$ to $V_{\varepsilon}^{\prime}$ (dual of $V_{\varepsilon}$ ) with $P_{\varepsilon}^{\star}$ given for every $g \in H^{-1}(\Omega)$ by

$$
\forall v \in V_{\varepsilon},\left\langle P_{\varepsilon}^{\star} g, v\right\rangle_{V_{\varepsilon}^{\prime}, V_{\varepsilon}}=\left\langle g, P_{\varepsilon} v\right\rangle_{H^{-1}(\Omega), H_{0}^{1}(\Omega)} .
$$

Definition 2.2 ([5]) Let $A^{\varepsilon} \in M(\alpha, \beta ; \Omega)$ and $T_{\varepsilon}$ be admissible in $\Omega$. We say that the pair $\left(A^{\varepsilon}, T_{\varepsilon}\right) H^{0}$ converges to the matrix $A^{0} \in M\left(\alpha^{\prime}, \beta^{\prime} ; \Omega\right)$ and we write $\left(A^{\varepsilon}, T_{\varepsilon}\right) \stackrel{H^{0}}{\rightarrow} A^{0}$ in $\Omega$ if and only if for every function $g$ of $L^{2}(\Omega)$, and every subsequence of $\varepsilon$ (still denoted by $\varepsilon$ ) such that $\chi^{\varepsilon} \rightarrow \chi^{0}$ weakly ${ }^{\star}$ in $L^{\infty}(\Omega)$ ( $\chi^{0}$ depending upon the subsequence), the solution $v^{\varepsilon}$ of

$$
\left\{\begin{array}{l}
-\operatorname{div}\left(A^{\varepsilon} \nabla v^{\varepsilon}\right)=g \text { in } \Omega_{\varepsilon} \\
\left(A^{\varepsilon} \nabla v^{\varepsilon}\right) \cdot v=0 \text { on } \partial T_{\varepsilon} \\
v^{\varepsilon}=0 \text { on } \partial \Omega
\end{array}\right.
$$

satisfies the weak convergence

$$
\left\{\begin{array}{l}
\text { i) } P_{\varepsilon}\left(v^{\varepsilon}\right) \longrightarrow v^{0} \text { weakly in } H_{0}^{1}(\Omega) \\
\text { ii) } A^{\varepsilon} \overline{\nabla v^{\varepsilon}} \rightarrow A^{0} \nabla v^{0} \text { weakly in } L^{2}(\Omega)^{n}
\end{array}\right.
$$

where $v^{0}$ is the unique solution of the problem

$$
\left\{\begin{array}{l}
-\operatorname{div}\left(A^{0} \nabla v^{0}\right)=\chi^{0} g \text { in } \Omega \\
v^{0}=0 \text { on } \partial \Omega
\end{array}\right.
$$

Remark 2.3 (1) In [5] the definition of $H^{0}$-convergence is given for $f \in H^{-1}(\Omega)$. This latter and Definition 2.2 are equivalent in view of [5, Theorem 1.5].

(2) In the case of $T_{\varepsilon}=\emptyset$, this definition reduces to the definition of $H$-convergence.

The main properties of the $H^{0}$-convergence are given by the results below.

Theorem 2.4 (Compactness [5]) Let $A^{\varepsilon} \in M(\alpha, \beta ; \Omega)$ and $T_{\varepsilon}$ be admissible in $\Omega$. Then, there exists a subsequence of $\{\varepsilon\}$ (still denoted by $\{\varepsilon\})$ and a matrix $A^{0} \in M\left(\frac{\alpha}{C^{2}}, \beta ; \Omega\right)$ such that $\left\{\left(A^{\varepsilon}, T_{\varepsilon}\right)\right\} H^{0}$-converges to $A^{0}$.

Proposition 2.5 [5] The pair $\left(A^{\varepsilon}, T_{\varepsilon}\right) H^{0}$-converges to $A^{0}$ if and only if $\left({ }^{t} A^{\varepsilon}, T_{\varepsilon}\right) H^{0}$-converges to ${ }^{t} A^{0}$. 
Finally, we complete the preliminary results by giving a pointwise estimate of the dierence of two $H^{0}$-limits. This result needs the following lemma (which is a directly consequence of [5, Proposition 1.14]):

Lemma 2.6 Assume that $\left(A^{\varepsilon}, T_{\varepsilon}\right) \stackrel{H^{0}}{\rightarrow} A^{0}$ in $\Omega$ and suppose that for every $\lambda \in \mathbb{R}^{n \times n}$, there exists a sequence $\left\{v_{\lambda}^{\varepsilon}\right\}$ bounded in $H^{1}(\Omega)$ such that

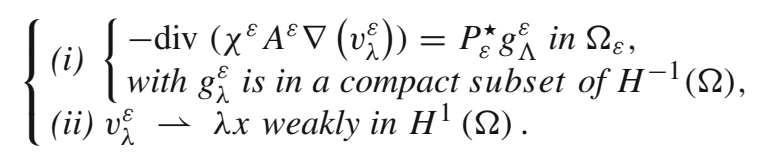

Then, if we set

$$
N^{\varepsilon} \lambda=\nabla v_{\lambda}^{\varepsilon}, \forall \lambda \in \mathbb{R}^{n},
$$

we will have $\chi^{\varepsilon} A^{\varepsilon} N^{\varepsilon} \rightarrow A^{0} \lambda$ weakly in $L^{2}(\Omega)^{n}$ and $N^{\varepsilon}$ is a corrector for the pair $\left(A^{\varepsilon}, T_{\varepsilon}\right)$ in the sense that

$$
\lim _{\varepsilon \rightarrow 0}\left\|\nabla v^{\varepsilon}-N^{\varepsilon} v^{0}\right\|_{L^{1}\left(\Omega_{\varepsilon}\right)^{n}}=0
$$

where $v^{\varepsilon}$ and $v^{0}$ are solutions of (2.1) and (2.3) respectively.

We are now able to give a pointwise estimate of the dierence of two $H^{0}$-limits.

Theorem 2.7 Let $T_{\varepsilon}^{1}$ and $T_{\varepsilon}^{2}$ be admissible in $\Omega, A_{1}^{\varepsilon} \in \mathcal{M}(\alpha, \beta ; \Omega)$ and $A_{2}^{\varepsilon} \in \mathcal{M}\left(\alpha^{\prime}, \beta^{\prime} ; \Omega\right)$ such that

$$
\left\{\begin{array}{l}
\left(A_{1}^{\varepsilon}, T_{\varepsilon}^{1}\right) \stackrel{H^{0}}{\rightarrow} A_{1}^{0} \quad \text { in } \Omega, \\
\left(A_{2}^{\varepsilon}, T_{\varepsilon}^{2}\right) \stackrel{H^{0}}{\rightarrow} A_{2}^{0} \quad \text { in } \Omega, \\
\chi_{2}^{\varepsilon}\left|A_{1}^{\varepsilon}(x)-A_{2}^{\varepsilon}(x)\right| \leq h^{\varepsilon}(x) \text { a.e. in } \Omega, \\
\text { with } h^{\varepsilon} \longrightarrow h^{0} \quad \text { strongly in } L^{1}(\Omega) .
\end{array}\right.
$$

\section{Assume that}

(i) $\chi_{1}^{\varepsilon}-\chi_{2}^{\varepsilon} \rightarrow 0$ strongly in $L^{1}(\Omega)$,

(ii) $\left(A_{1}^{\varepsilon}, T_{\varepsilon}^{1}\right)$ admits a corrector satisfying (2.4)-(2.5),

(iii) $\left(A_{2}^{\varepsilon}, T_{\varepsilon}^{2}\right)$ admits a corrector $N^{\varepsilon}$ satisfying (2.4)-(2.5) and

$$
\left\{\begin{array}{l}
\exists p>2, \text { such that }\left\|N^{\varepsilon}\right\|_{L^{p}(\Omega)^{n \times n}} \leq \rho \\
\text { with } \rho>0 \text { is independent of } \varepsilon
\end{array}\right.
$$

Then,

$$
\left|A_{1}^{0}-A_{2}^{0}\right| \leq \sqrt{\frac{\beta \beta^{\prime}}{\alpha \alpha^{\prime}}} h^{0} \quad \text { a.e. in } \Omega .
$$

Proof The proof is obtained by using Lemma 2.6 and Proposition 2.5, and by following the same techniques used to prove a similar result given for the elasticity case in [11, Theorem 28].

\section{Remark 2.8 Assumptions}

(i)-(iii) of Theorem 2.7 are reasonable. Indeed,

-(i) is obviously satisfied when $T_{\varepsilon}^{1}=T_{\varepsilon}^{2}$ for every $\varepsilon$,

-(ii) is satisfied when there exists a bounded domain $O$ in $\mathbb{R}^{n}$ in which $\Omega$ is relatively compact and for which $T_{\varepsilon}$ is admissible (see the proof of [5, Proposition 1.15]),

-(iii) is satisfied for the classical periodic case and also for the non-periodic case considered in [5]. 


\section{Statement of compactness results}

In this section, we give our compactness results for the $H^{0}$-convergence of a class of elliptic and uniformly equicontinuous operators in perforated domains. Firstly, we introduce the set $\mathcal{M}_{E q u i}(\alpha, \beta, \omega ; \Omega)$ in the following definition :

Definition 3.1 For two real numbers $\alpha, \beta$ such that $0<\alpha<\beta$ and $\omega$ a function defined from $\mathbb{R}^{+}$to $\mathbb{R}^{+}$ nondecreasing and continuous at $0, \mathcal{M}_{E q u i}(\alpha, \beta, \omega ; \Omega)$ denotes the set of all Caratheodory functions

$$
A:(x, d) \in(\Omega, \mathbb{R}) \longmapsto A(x, d) \in \mathbb{R}^{n \times n}
$$

satisfying the following assumptions:

(i) for every $d \in \mathbb{R}, \quad A(d) \doteq A(\cdot, d) \in \mathcal{M}(\alpha, \beta ; \Omega)$,

(ii) for almost every $x$ in $\Omega$ and for every $d, d^{\prime} \in \mathbb{R}$, one has

$$
\left|A(x, d)-A\left(x, d^{\prime}\right)\right| \leq \omega\left(\left|d-d^{\prime}\right|\right) .
$$

Our first main result is the following:

Theorem 3.2 Let $\left\{T_{\varepsilon}\right\}$ be a sequence admissible in $\Omega$ and $\left\{A^{\varepsilon}\right\}$ be a sequence of elements of $\mathcal{M}_{E q u i}(\alpha, \beta, \omega ; \Omega)$. Assume that $\omega(0)=0$ and

$$
\left\{\begin{array}{l}
\forall d \in \mathbb{R}, \exists p>2 \text { s.t. }\left(A^{\varepsilon}(d), T_{\varepsilon}\right) \text { admits a corrector which } \\
\text { satisfies (2.4)-(2.5) and is bounded in } L^{p}(\Omega) \text { independently of } \varepsilon .
\end{array}\right.
$$

Then, there exists a subsequence of $\{\varepsilon\}$ (still denoted by $\{\varepsilon\}$ ), and an element $A^{0} \in \mathcal{M}_{E q u i}\left(\frac{\alpha}{C^{2}}, \beta, \frac{\beta}{\alpha} \omega ; \Omega\right.$ ) such that

$$
\left(A^{\varepsilon}(d), T_{\varepsilon}\right) \stackrel{H^{0}}{\rightarrow} A^{0}(d) \text { in } \Omega, \quad \forall d \in \mathbb{R} .
$$

Moreover, if we suppose that there exists a bounded domain $O$ in $\mathbb{R}^{n}$ in which $\Omega$ is relatively compact and for which $T_{\varepsilon}$ is also admissible, we have

$$
\left(A^{\varepsilon}(v), T_{\varepsilon}\right) \stackrel{H^{0}}{\rightarrow} A^{0}(v) \text { in } \Omega, \forall v \in L^{1}(\Omega) .
$$

Remark 3.3 (i) A similar property to (3.2) is given in [14] in the case of fixed domain when the mapping $d \rightarrow A^{\varepsilon}(\cdot, d)$ is of class $C^{k}$ (or real analytic) from an open set $D$ of $\mathbb{R}^{p}$ into $L^{\infty}\left(\Omega ; L\left(\mathbb{R}^{n} ; \mathbb{R}^{n}\right)\right.$ ) for every $p \in \mathbb{N}^{*}$.

(ii) Theorem 3.2 still holds if $d \in \mathbb{R}^{p}$ and $v \in L^{1}(\Omega)^{p}$ for every $p \in \mathbb{N}^{*}$.

As a consequence of Theorem 3.2, we obtained a general homogenization result for some quasilinear equations in perforated domain beyond periodic setting.

Theorem 3.4 Let $\left\{T_{\varepsilon}\right\}$ be a sequence admissible in $\Omega$ and suppose that there exists a bounded domain $O$ in $\mathbb{R}^{n}$ in which $\Omega$ is relatively compact and for which $T_{\varepsilon}$ is also admissible. Let $\left\{A^{\varepsilon}\right\}$ be a sequence in $\mathcal{M}_{\text {Equi }}(\alpha, \beta, \omega ; \Omega)$ which satisfies (3.1). Assume that $\omega$ is continuous with $\omega(d)>0 \forall d>0$ and

$$
\text { for any } r>0, \quad \lim _{s \rightarrow 0} \int_{s}^{r} \frac{\mathrm{d} t}{\omega(t)}=+\infty .
$$

Then, there exists subsequence of $\{\varepsilon\}$ (still denoted by $\{\varepsilon\}$ ) with $\chi^{\varepsilon}$ converges to a some $\chi^{0}$ weakly ${ }^{\star}$ in $L^{\infty}(\Omega)$, such that for every function $f$ of $L^{2}(\Omega)$, the (unique) solution $u^{\varepsilon}$ of the problem:

$$
\left\{\begin{array}{l}
-\operatorname{div}\left(A^{\varepsilon}\left(u^{\varepsilon}\right) \nabla u^{\varepsilon}\right)=f \text { in } \Omega_{\varepsilon} \\
A^{\varepsilon}\left(u^{\varepsilon}\right) \nabla u^{\varepsilon} \cdot v=0 \text { on } \partial T_{\varepsilon} \\
u^{\varepsilon}=0 \text { on } \partial \Omega
\end{array}\right.
$$


satisfies

$$
\left\{\begin{array}{l}
\text { (i) } P_{\varepsilon}\left(u^{\varepsilon}\right) \rightarrow u^{0} \text { weakly in } H_{0}^{1}(\Omega), \\
\text { (ii) } u^{\varepsilon} \rightarrow \chi^{0} u^{0} \text { weakly in } L^{2}(\Omega), \\
\text { (iii) } A^{\varepsilon}\left(u^{\varepsilon}\right) \nabla u^{\varepsilon} \rightarrow A^{0}\left(u^{0}\right) \nabla u^{0} \text { weakly in } L^{2}(\Omega)^{n},
\end{array}\right.
$$

where $u^{0}$ is the (unique) solution of

$$
\left\{\begin{array}{l}
-\operatorname{div}\left(A^{0}\left(u^{0}\right) \nabla u^{0}\right)=\chi^{0} f \quad \text { in } \Omega \\
u^{0}=0 \text { on } \partial \Omega
\end{array}\right.
$$

with $A^{0}$ the family of matrices given by Theorem 3.2.

Remark 3.5 Assumption (3.4) introduced initially in [9] implies that $\lim _{d \rightarrow 0} \omega(d)=0$. If this assumption is replaced by just the fact that $\lim _{d \rightarrow 0} \omega(d)=0$, the uniqueness will no longer be guaranteed for the solutions of (3.5) and (3.7).

\section{Proofs of compactness results}

We give in this section the proofs of our main results. The proofs are an adaptation of the similar ones given in [4] for fixed domains.

Proof of Theorem 3.2 We give the proof in two steps.

Step 1. Let us prove that there exists $A^{0} \in \mathcal{M}_{E q u i}\left(\frac{\alpha}{C^{2}}, \beta, \frac{\beta}{\alpha} \omega ; \Omega\right)$ which satisfies convergence (3.2) up to subsequence. Using Theorem 2.4 and the diagonal subsequence procedure, we extract a subsequence of $\{\varepsilon\}$ (still denoted by $\{\varepsilon\}$ ) such that, for every $d \in \mathbb{Q}$, we will have

$$
\left(A^{\varepsilon}(d), T_{\varepsilon}\right) H^{0} \text {-converges to a limit } A^{0}(d) \in \mathcal{M}\left(\frac{\alpha}{C^{2}}, \beta ; \Omega\right) .
$$

Hence, by the fact that $A^{\varepsilon} \in \mathcal{M}_{E q u i}(\alpha, \beta, \omega ; \Omega)$, Assumption (3.1) and Theorem 2.7, we obtain

$$
\left|A^{0}(x, d)-A^{0}\left(x, d^{\prime}\right)\right| \leq \frac{\beta}{\alpha} \omega\left(\left|d-d^{\prime}\right|\right) \quad \text { a.e. } x \in \Omega, \forall d, d^{\prime} \in \mathbb{Q} .
$$

Thus, the mapping

$$
\begin{aligned}
A^{0}: \mathbb{Q} & \longrightarrow \mathbb{L}^{\infty}(\Omega)^{n \times n}, \\
d & \longmapsto A^{0}(d)
\end{aligned}
$$

is uniformly continuous. Hence, it is extensible to a mapping (denoted again by $A^{0}$ ) defined and uniformly continuous on all $\mathbb{R}$ (since $\mathbb{Q}$ is dense in $\mathbb{R}$ ), namely

$$
\left|A^{0}(x, d)-A^{0}\left(x, d^{\prime}\right)\right| \leq \frac{\beta}{\alpha} \omega\left(\left|d-d^{\prime}\right|\right), \quad \text { a.e. } x \in \Omega, \forall d, d^{\prime} \in \mathbb{R} .
$$

On the other hand, let $d \in \mathbb{R}$ and $\left\{d_{m}\right\}$ be a sequence in $\mathbb{Q}$ which converges to $d$ as $m \rightarrow \infty$. Thanks to Theorem 2.4, there exists a subsequence of $\{\varepsilon\}$ (still denoted by $\{\varepsilon\}$ ) such that

$$
\left(A^{\varepsilon}(d), T_{\varepsilon}\right) H^{0} \text {-converges to some } A \in \mathcal{M}\left(\frac{\alpha}{C^{2}}, \beta ; \Omega\right) .
$$

Since, for every $\varepsilon>0$, we have

$$
\left|A^{\varepsilon}(x, d)-A^{\varepsilon}\left(x, d_{m}\right)\right| \leq \omega\left(\left|d-d_{m}\right|\right), \quad \text { a.e. } x \in \Omega,
$$

then from this, (4.1), (4.3), Assumption (3.1) and Theorem 2.7, it comes

$$
\left|A(x)-A^{0}\left(x, d_{m}\right)\right| \leq \frac{\beta}{\alpha} \omega\left(\left|d-d_{m}\right|\right), \quad \text { a.e. } x \in \Omega .
$$


This, with (4.2) and by the triangle inequality, we deduce that for almost every $x$ in $\Omega$

$$
\begin{aligned}
\left|A(x)-A^{0}(x, d)\right| & \leq\left|A(x)-A^{0}\left(x, d_{m}\right)\right|+\left|A^{0}(x, d)-A^{0}\left(x, d_{m}\right)\right| \\
& \leq 2 \frac{\beta}{\alpha} \omega\left(\left|d-d_{m}\right|\right) .
\end{aligned}
$$

Using the continuity of $\omega$ at 0 , passing to the limit in this inequality as $m \rightarrow \infty$, we find

$$
A(x)=A^{0}(x, d), \quad \text { a.e. } x \in \Omega .
$$

Step 2. We now show property (3.3). Let $v \in L^{1}(\Omega)$. Then, $A^{\varepsilon}(v(\cdot)) \doteq A^{\varepsilon}(\cdot, v(\cdot))$ belongs to $\mathcal{M}(\alpha, \beta ; \Omega)$. Hence, taking into account Theorem 2.4, there exists $B^{0} \in \mathcal{M}\left(\frac{\alpha}{C^{2}}, \beta ; \Omega\right)$ such that to up a subsequence, we have

$$
\left(A^{\varepsilon}(v), T_{\varepsilon}\right) \stackrel{H^{0}}{\rightarrow} B^{0} .
$$

On the other hand, since $v \in L^{1}(\Omega)$, there exists a sequence of step functions $\left\{v^{m}\right\}$ such that $v^{m} \rightarrow v$ strongly in $L^{1}(\Omega)$, and $v^{m}$ is of the form

$$
v^{m}=\sum_{i=1}^{i=k} l_{i}^{m} \chi_{Y_{i}}, \quad \text { a.e. in } \Omega,
$$

where $\left\{Y_{i}\right\}_{1 \leq i \leq k}$ is a family of disjoint rectangles of $\mathbb{R}^{n}$ included in $\Omega$ and $l_{i}^{m}$ real constants. Set

$$
\left\{\begin{array}{l}
Y_{0}=\Omega \backslash \cup \underset{1 \leq i \leq k}{\cup} \overline{Y_{i}} \\
\chi_{i}=\chi_{Y_{i}} \\
\chi_{0}=\chi_{Y_{0}}
\end{array}\right.
$$

We have

$$
\left\{\begin{array}{l}
\forall i \in\{1, \ldots, k\},\left|A^{\varepsilon}(x, v(x))-A^{\varepsilon}\left(x, l_{i}^{m}\right)\right| \leq \omega\left(\left|v(x)-l_{i}^{m}\right|\right) \quad \text { a.e. in } \Omega, \\
\left|A^{\varepsilon}(x, v(x))-A^{\varepsilon}(x, 0)\right| \leq \omega(|v(x)-0|) \text { a.e. in } \Omega,
\end{array}\right.
$$

and (3.2) gives

$$
\left\{\begin{array}{l}
A^{\varepsilon}\left(l_{i}^{m}, T_{\varepsilon}\right) \stackrel{H^{0}}{\rightarrow} A^{0}\left(l_{i}^{m}\right) \\
A^{\varepsilon}\left((0), T_{\varepsilon}\right) \stackrel{H^{0}}{\rightarrow} A^{0}(0) .
\end{array}\right.
$$

Hence, using (4.4), (4.6), (4.7), Assumption (3.1), point (ii) of Remark 2.8 and by Theorem 2.7, we obtain

$$
\left\{\begin{array}{l}
\forall i \in\{1, \ldots, k\},\left|B^{0}(x)-A^{0}\left(x, l_{i}^{m}\right)\right| \leq \frac{\beta}{\alpha} \omega\left(\left|v(x)-l_{i}^{m}\right|\right) \quad \text { a.e. in } \Omega, \\
\left|B^{0}(x)-A^{0}(x, 0)\right| \leq \frac{\beta}{\alpha} \omega(|v(x)-0|) \quad \text { a.e. } i n \Omega,
\end{array}\right.
$$

which implies that for almost every $x$ in $\Omega$

$$
\begin{aligned}
\left|B^{0}(x)-A^{0}\left(x, v^{m}\right)\right| & =\left|B^{0}(x)-\sum_{i=1}^{i=k} A^{0}\left(x, l_{i}^{m}\right) \chi_{i}(x)+A^{0}(x, 0) \chi_{0}(x)\right| \\
& \leq \sum_{i=1}^{i=k} \chi_{i}(x) \frac{\beta}{\alpha} \omega\left(\left|v(x)-l_{i}^{m}\right|\right)+\chi_{0}(x) \frac{\beta}{\alpha} \omega(|v(x)-0|) \\
& =\frac{\beta}{\alpha} \omega\left(\left|v(x)-v^{m}(x)\right|\right) .
\end{aligned}
$$

Moreover, thanks to (4.2), we have

$$
\left|A^{0}(x, v(x))-A^{0}\left(x, v^{m}(x)\right)\right| \leq \frac{\beta}{\alpha} \omega\left(\left|v(x)-v^{m}(x)\right|\right) \quad \text { a.e. in } \Omega .
$$


Hence, from this two latter inequalities, it follows from triangle inequality that

$$
\begin{aligned}
\left|B^{0}(x)-A^{0}(x, v(x))\right| & \leq\left|B^{0}(x)-A^{0}\left(x, v^{m}(x)\right)\right|+\left|A^{0}\left(x, v^{m}(x)\right)-A^{0}(x, v(x))\right| \\
& \leq 2 \frac{\beta}{\alpha} \omega\left(\left|v(x)-v^{m}(x)\right|\right) \text { a.e. in } \Omega .
\end{aligned}
$$

Since $\omega$ is continuous at 0 , passing to the limit in this inequality when $m \rightarrow \infty$, one obtains

$$
B^{0}(x)=A^{0}(x, v(x)) \text { a.e. in } \Omega,
$$

which, with (4.4), gives ( 3.3).

Proof of Theorem 3.4 First, note that problem (3.5) (respect. (3.7) has a unique solution in $H_{0}^{1}\left(\Omega_{\varepsilon}\right)$ (respect. $H_{0}^{1}(\Omega)$ ) thanks to [6].

Second, taking $u^{\varepsilon}$ as a test function in the variational formulation of (3.5), we obtain

$$
\left\|P_{\varepsilon} u^{\varepsilon}\right\|_{H_{0}^{1}(\Omega)} \leq C\left\|u^{\varepsilon}\right\|_{H_{0}^{1}\left(\Omega_{\varepsilon}\right)} \leq \frac{C}{\alpha}\|f\|_{L^{2}(\Omega \varepsilon)} .
$$

Hence, we can extract a subsequence of $\{\varepsilon\}$ (still denoted by $\{\varepsilon\}$ ), such that

$$
P_{\varepsilon} u^{\varepsilon} \rightarrow u^{0} \text { weakly in } H_{0}^{1}(\Omega),
$$

hence

$$
P_{\varepsilon} u^{\varepsilon} \rightarrow u^{0} \text { strongly in } L^{2}(\Omega) \text {. }
$$

This implies, for every $m$, that

$$
P_{\varepsilon} u^{\varepsilon}-v^{m} \rightarrow u^{0}-v^{m} \quad \text { strongly in } L^{2}(\Omega),
$$

where $\left\{v^{m}\right\}$ is a sequence of functions introduced in (4.5) such that $v^{m} \rightarrow u^{0}$ strongly in $L^{1}(\mathbb{R})$. So, thanks to continuity of $\omega$, we get

$$
\omega\left(\left|P_{\varepsilon} u^{\varepsilon}-v^{m}\right|\right) \rightarrow \omega\left(\left|u^{0}-v^{m}\right|\right) \quad \text { strongly in } L^{1}(\Omega) .
$$

On the other hand, since $A^{\varepsilon}\left(P_{\varepsilon} u^{\varepsilon}\right) \doteq A^{\varepsilon}\left(\cdot, P_{\varepsilon} u^{\varepsilon}(\cdot)\right) \in \mathcal{M}(\alpha, \beta ; \Omega)$, there exists a subsequence of $\{\varepsilon\}$ (still denoted by $\{\varepsilon\})$ and $C^{0} \in \mathcal{M}\left(\frac{\alpha}{C^{2}}, \beta ; \Omega\right)$, such that

$$
\left(A^{\varepsilon}\left(P_{\varepsilon} u^{\varepsilon}\right), T_{\varepsilon}\right) \stackrel{H^{0}}{\rightarrow} C^{0},
$$

but

$$
\forall \varepsilon>0,\left|A^{\varepsilon}\left(x, P_{\varepsilon} u^{\varepsilon}(x)\right)-A^{\varepsilon}\left(x, v^{m}(x)\right)\right| \leq \omega\left(\left|P_{\varepsilon} u^{\varepsilon}(x)-v^{m}(x)\right|\right) \text {, a.e. in } \Omega,
$$

hence by this last inequality, (4.8), (4.9), Theorem 3.2, Assumption (3.1), point (ii) of Remark 2.8 and Theorem 2.7 , it comes

$$
\left|C^{0}(x)-A^{0}\left(x, v^{m}(x)\right)\right| \leq \frac{\beta}{\alpha} \omega\left(\left|u^{0}(x)-v^{m}(x)\right|\right), \text { a.e. in } \Omega .
$$

This gives

$$
\begin{aligned}
\left|C^{0}(x)-A^{0}\left(x, u^{0}(x)\right)\right| & \leq\left|C^{0}(x)-A^{0}\left(x, v^{m}(x)\right)\right|+\left|A^{0}\left(x, v^{m}(x)\right)-A^{0}\left(x, u^{0}(x)\right)\right| \\
& \leq 2 \frac{\beta}{\alpha} \omega\left(\left|u^{0}(x)-v^{m}(x)\right|\right) \quad \text { a.e. in } \Omega .
\end{aligned}
$$

Since $\lim _{d \rightarrow 0} \omega(d)=0$, passing to the limit in this inequality when $m \rightarrow \infty$, we obtain

$$
C^{0}(x)=A^{0}\left(x, u^{0}(x)\right) \quad \text { a.e. in } \Omega .
$$

Then, from this and (4.9), we find

$$
\left(A^{\varepsilon}\left(P_{\varepsilon} u^{\varepsilon}\right), T_{\varepsilon}\right) \stackrel{H^{0}}{\rightarrow} A^{0}\left(\cdot, u^{0}(\cdot)\right),
$$


which implies by the definition of the $H^{0}$-convergence and the uniqueness of the solutions of (3.5) and (3.7) that

$$
\left\{\begin{array}{l}
P_{\varepsilon} u^{\varepsilon} \rightarrow u^{0} \text { weakly in } H_{0}^{1}(\Omega), \\
A^{\varepsilon}\left(u^{\varepsilon}\right) \nabla u^{\varepsilon} \rightarrow A^{0}\left(u^{0}\right) \nabla u^{0} \text { weakly in } L^{2}(\Omega)^{n},
\end{array}\right.
$$

where $u^{0}$ is the solution of (3.7), which completes proof of (i) and (iii) of (3.6).

Finally, we deduce (3.6)ii) from the fact that

$$
\chi^{\varepsilon} \rightarrow \chi^{0} \text { weakly * in } L^{\infty}(\Omega)
$$

and

$$
P_{\varepsilon} u^{\varepsilon} \rightarrow u^{0} \text { strongly in } L^{2}(\Omega)
$$

\section{Applications}

As an application of our results, we consider the classical periodic case and a non-periodic case.

Let $\theta$ a diffeomorphism of class $C^{2}$ from $\mathbb{R}^{n}$ onto $\mathbb{R}^{n}$ and introduce the holes $T_{\varepsilon}$ defined by

$$
\left\{\begin{array}{l}
T_{\varepsilon}=\underset{k \in \mathbb{Z}^{n}}{\cup}\left\{S_{\varepsilon}^{k} \text { s.t. } S_{\varepsilon}^{k} \subset \Omega, \operatorname{dist}(\theta(k \varepsilon), \partial \Omega)>2 \varepsilon\right\}, \\
\text { with } S_{\varepsilon}^{k}=\left\{x \in \mathbb{R}^{n} \text { s.t. }|x-\theta(k \varepsilon)| \leq \delta \varepsilon\right\}, k \in \mathbb{Z}^{n},
\end{array}\right.
$$

where $\delta \in] 0,1]$. Let $Y=\left[-\frac{1}{2}, \frac{1}{2}\right]^{n}$ and set

$$
\left.A^{\varepsilon}(x, d)\right)=A\left(\frac{\theta^{-1}(x)}{\varepsilon}\right)
$$

with $A \in \mathcal{M}_{E q u i}(\alpha, \beta, \omega ; Y)$. Assume that $\omega$ is continous with $\omega(d)>0 \forall d>0$ and

$$
\text { for any } r>0, \quad \lim _{s \rightarrow 0} \int_{s}^{r} \frac{\mathrm{d} t}{\omega(t)}=+\infty .
$$

In what follows, the spherical geometry of the holes can be generalized to the case where a regular boundary hole with a finite number of connected components replace a ball.

\subsection{Classical periodic case}

Take here $\theta=I d_{\mathbb{R}^{n}}$ and $\delta=\frac{1}{3}$. Then, the pair $\left(A^{\varepsilon}(\cdot, \cdot), T_{\varepsilon}\right)$ satisfies all assumptions of Theorem 3.4 and it is well-known that in this case (see [10])

$$
\left\{\begin{array}{l}
\forall d \in \mathbb{R},\left(A^{\varepsilon}(\cdot, d), T_{\varepsilon}\right) \stackrel{H^{0}}{\rightarrow} A^{0}(d), \\
\chi^{\varepsilon} \rightarrow \frac{\left|Y^{*}\right|}{|Y|} \text { weakly }{ }^{\star} \text { in } L^{\infty}(\Omega)
\end{array}\right.
$$

with $A^{0}(d)$ is independent of $x$ and given by

$$
\forall \lambda \in \mathbb{R}^{n}, \quad A^{0}(d) \lambda=\frac{1}{|Y|} \int_{Y^{\star}} A(y, d) \nabla_{y} v_{\lambda}(y, d) \mathrm{d} y,
$$

where

$$
Y^{*}=Y \backslash T, \quad T=\left\{x \in \mathbb{R}^{n} \quad \text { s.t. }|x| \leq \frac{1}{3}\right\},
$$


and for all $\lambda \in \mathbb{R}^{n}, y \longmapsto v_{\lambda}(y, d)$ be the solution of

$$
\left\{\begin{array}{l}
\left.-\operatorname{div}\left(A(y, d) \nabla v_{\lambda}(y, d)\right)\right)=0 \text { in } Y^{\star} \\
\left(A(y, d) \nabla v_{\lambda}(y, d) \nabla u^{\varepsilon} \cdot v=0 \text { on } \partial T\right. \\
v_{\lambda}(y, d)-\lambda \cdot y \text { is } Y-\text { periodic with mean value } 0 .
\end{array}\right.
$$

In this framework, we have the following result about the convergence of problem (3.5):

Proposition 5.1 For every $f \in L^{2}(\Omega)$, the solution $u^{\varepsilon}$ of problem (3.5) satisfies

$$
\left\{\begin{array}{l}
\text { (i) } P_{\mathcal{E}}\left(u^{\varepsilon}\right) \rightarrow u^{0} \text { weakly in } H_{0}^{1}(\Omega), \\
\text { (ii) } \widetilde{u}^{\varepsilon} \overrightarrow{\chi^{0}} u^{0} \text { weakly in } L^{2}(\Omega), \\
\text { (iii) } A^{\varepsilon}\left(u^{\varepsilon}\right) \nabla u^{\varepsilon} \rightarrow A^{0}\left(u^{0}\right) \nabla u^{0} \quad \text { weakly in } L^{2}(\Omega)^{n},
\end{array}\right.
$$

where $u^{0}$ is the solution of

$$
\left\{\begin{array}{l}
-\operatorname{div}\left(A^{0}\left(u^{0}\right) \nabla u^{0}\right)=\chi^{0} f \quad \text { in } \Omega, \\
u^{0}=0 \text { on } \partial \Omega .
\end{array}\right.
$$

and where $A^{0}$ defined by (5.1) belongs to $\mathcal{M}_{E q u i}\left(\frac{\alpha}{C^{2}}, \beta, \frac{\beta}{\alpha} \omega ; \Omega\right)$.

Remark 5.2 In the geometric framework of this example, Proposition 5.1 coincides with a result given in [7] by using the periodic unfolding, when the nonlinear Robin boundary condition on the holes reduces to the homogeneous Neumann condition.

\subsection{Non-periodic case}

Consider here the non-periodic perforated domain introduced in [5, Section 3] when studying the corresponding linear case. We suppose that $\theta^{-1}$ has a Lipschitz constant $\kappa^{-1}$ with $\kappa>2$ and take $\delta=1$. In this case, from [5, Sections 3-4], we deduce easily that for every $d \in \mathbb{R}$, the pair $\left(A^{\varepsilon}, T_{\varepsilon}\right)$ satisfies all assumptions of Theorem 3.4 and

$$
\left\{\begin{array}{l}
\left.\forall d \in \mathbb{R},\left(A^{\varepsilon}(\cdot, d), T_{\varepsilon}\right)\right) \stackrel{H^{0}}{\rightarrow} A^{0}(\cdot, d) \text { in } \Omega, \\
\chi^{\varepsilon}(\cdot) \rightarrow \frac{\left|Y^{*}(\cdot)\right|}{|Y(\cdot)|} \text { weakly * in } L^{\infty}(\Omega),
\end{array}\right.
$$

with

$$
A^{0}(x, d)=B_{x}^{0}(d)
$$

where $B_{x}^{0}(d)$ is defined by

$$
\forall \lambda \in \mathbb{R}^{n}, \quad B_{x}^{0}(d) \lambda=\frac{1}{|Y(x)|} \int_{Y(x)^{\star}} B(x, y, d) \nabla_{y} v_{\lambda}(x, y, d) \mathrm{d} y,
$$

and where we have

$$
\left\{\begin{array}{l}
B(x, y, d)=A\left(\left[\nabla \theta\left(\theta^{-1}(x)\right)\right]^{-1} y, d\right) \\
Y(x)=\left\{\nabla \theta\left(\theta^{-1}(x)\right) z \text { s.t. } \quad z \in Y\right\} \\
T_{1}=\left\{z \in \mathbb{R}^{n} \text { s.t. }|z| \leq 1\right\} \\
Y(x)^{\star}=Y(x) \backslash T_{1}
\end{array}\right.
$$

and for all $\lambda \in \mathbb{R}^{n}, y \longmapsto v_{\lambda}(x, y, d)$ be the solution of

$$
\left\{\begin{array}{l}
\left.-\operatorname{div}\left(B(x, y, d) \nabla v_{\lambda}(x, y, d)\right)\right)=0 \text { in } Y(x)^{\star} \\
\left(B(x, y, d) \nabla v_{\lambda}(x, y, d) \cdot v=0 \text { on } \partial T_{1}\right. \\
v_{\lambda}(x, y, d)-\lambda \cdot y \text { is } Y(x)-\text { periodic with mean value } 0 .
\end{array}\right.
$$

In this framework, we have the following result about the convergence of problem (3.5): 
Proposition 5.3 For every $f \in L^{2}(\Omega)$, the solution $u^{\varepsilon}$ of problem (3.5) satisfies

$$
\left\{\begin{array}{l}
\text { (i) } P_{\varepsilon}\left(u^{\varepsilon}\right) \rightarrow u^{0} \text { weakly in } H_{0}^{1}(\Omega), \\
\text { (ii) } u^{\varepsilon}=\chi^{0} u^{0} \text { weakly in } L^{2}(\Omega), \\
\text { (iii) } A^{\varepsilon}\left(u^{\varepsilon}\right) \nabla u^{\varepsilon} \rightarrow B_{x}^{0}\left(u^{0}\right) \nabla u^{0} \text { weakly in } L^{2}(\Omega)^{n},
\end{array}\right.
$$

where $u^{0}$ is the solution of

$$
\left\{\begin{array}{l}
-\operatorname{div}\left(B_{x}^{0}\left(u^{0}(x)\right) \nabla u^{0}(x)\right)=\frac{\left|Y^{*}(x)\right|}{|Y(x)|} f(x) \text { in } \Omega, \\
u^{0}(x)=0 \quad \text { on } \partial \Omega
\end{array}\right.
$$

and where $(x, d) \mapsto B_{x}^{0}(d)$ defined by (5.2) belongs to $\mathcal{M}_{E q u i}\left(\frac{\alpha}{C^{2}}, \beta, \frac{\beta}{\alpha} \omega ; \Omega\right)$.

Open Access This article is licensed under a Creative Commons Attribution 4.0 International License, which permits use, sharing, adaptation, distribution and reproduction in any medium or format, as long as you give appropriate credit to the original author(s) and the source, provide a link to the Creative Commons licence, and indicate if changes were made. The images or other third party material in this article are included in the article's Creative Commons licence, unless indicated otherwise in a credit line to the material. If material is not included in the article's Creative Commons licence and your intended use is not permitted by statutory regulation or exceeds the permitted use, you will need to obtain permission directly from the copyright holder. To view a copy of this licence, visit http://creativecommons.org/licenses/by/4.0/.

\section{References}

1. Artola, M.; Duvaut, G.: Homognisation d'une classe de problmes non linaires. C. R. Acad. Paris Ser. A 288, $775-778$ (1979)

2. Bendib, S.: Homogénéisation dune classe de problèmes non linéaires avec des conditions de Fourier dans des ouverts perforés. Thèse, Institut National Polytechnique de Lorraine (2004)

3. Bendib, S.; Tcheugou, T.R.: Homogénéisation dune classe de problèmes non linéaires dans des domaines perforés. C. R. Acad. Sci. Paris t. Ser. 1(328), 1145-1149 (1999)

4. Boccardo, L.; Murat, F.; Homogénéisation de problèmes quasi linéaires, Atti del convergno, Studio di Problemi-Limite dellAnalisi Funzionale (Bressanone 79 sett. 1981), vol. 1351. Pitagora Editrice. Bologna (1982)

5. Briane, M.; Damlamian, A.; Donato, P.: H-convergence in perforated domains, in non-linear partial differential equations and their applications. In: Cioranescu, D., Lions, J.L. (eds.) Collège de France seminar vol. XIII. Pitman Research Notes in Mathematics Series, vol. 391. Longman, New York (1998)

6. Cabarrubias, B.; Donato, P.: Existence and uniqueness for a quasilinear elliptic problem with nonlinear Robin conditions. Carpathian J. Math. 27(2), 173-184 (2011)

7. Cabarrubias, B.; Donato, P.: Homogenization of a quasilinear elliptic problem with nonlinear Robin boundary conditions. Appl. Anal. 91(6), 1111-1127 (2012)

8. Cardone, G.; Donato, P.; Gaudiello, A.: A compactness result for elliptic equations with subquadratic growth in perforated domains. Nonlinear Anal. Theor. Methods Appl. 32, 335-361 (1998)

9. Chipot, M.: Elliptic Equations: An Introductory Course, Birkhuser Advanced Texts: Basler Lehrbcher. Birkhuser Verlag, Basel (2009)

10. Cioranescu, D.; Saint Jean Paulin, J.: Homognisation in open sets with holes. J. Math. Anal. Appl. 71, 590-607 (1979)

11. Donato, P.; Haddadou, H.: Meyers type estimates in elasticity and applications to H-convergence. Adv. Math. Sci. Appl. 16(2), 537-567 (2006)

12. Murat, F.: H-convergence, Sminaire dAnalyse Fonctionnelle et Numrique, 1977/1978. Univ. dAlger, Multigraphed

13. Sango, M.: Homogenization of the Neumann problem for a quasilinear elliptic equation in a perforated domain. Netw Heterog. Media 5, 361-384 (2010)

14. Tartar, L.: The General Theory of Homogenization: A Personalized Introduction. Lecture Notes of the Unione Matematica Italiana, vol. 7. Springer, UMI, Berlin (2009)

Publisher's Note Springer Nature remains neutral with regard to jurisdictional claims in published maps and institutional affiliations. 